\title{
Research Paper \\ Relationship Between the Early Maladaptive Schema and Social Networks Addiction Among Semnan University of Medical Sciences Students
}

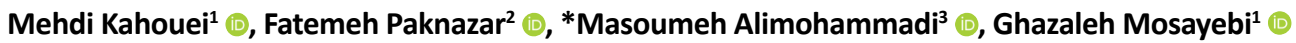

\author{
1. School of Allied Medical Sciences, Social Determinants of Health Research Center, Semnan University of Medical Sciences, Semnan, Iran. \\ 2. Social Determinants of Health Research Center, Semnan University of Medical Sciences, Semnan, Iran. \\ 3. Department of Psychology, Shahrood Branch, Islamic Azad University, Shahrood, Iran.
}

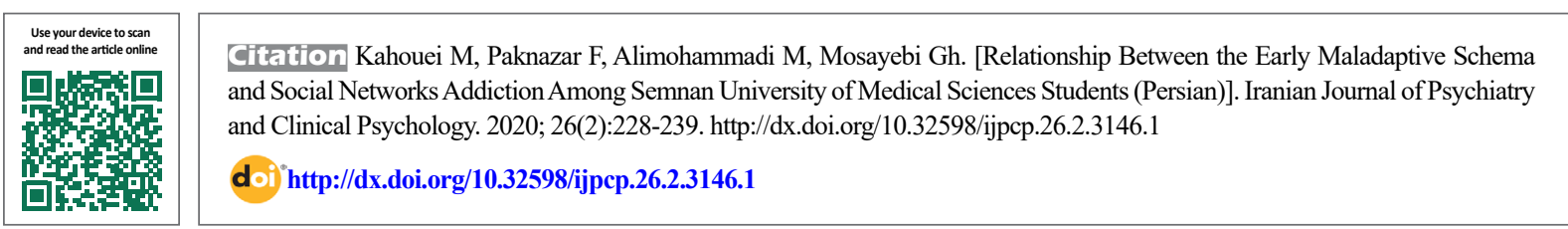

Received: 16 Jul 2019

Accepted: 02 Mar 2020

Available Online: $01 \mathrm{Jul} 2020$

Key words:

Early maladaptive

schema, Social net-

work sites, Addiction,

Students

\section{A B STRACT}

Objectives Early maladaptive schemas are self-destructive emotional and cognitive patterns that have been formed at the beginning of development and are repeated in life. Since early maladaptive schemas can be the most predictive of addiction and social networking addiction is also considered as an injury or complication. This study aimed to determine the relationship between early maladaptive schemas and social network addiction among students.

Methods This cross-sectional study was performed on 350 students of Semnan University of Medical Sciences. In this study, Yang's Short Form Questionnaire and the questionnaire of social network saddiction based on mobile were used. Data were analyzed by the Chi-square test and logistic regression.

Results There were 186 (53.2\%) students who had social network addiction. Of them, 215 (61.4\%) had at least one early maladaptive schema. The gender of the woman ( $\mathrm{OR}=0.35, \mathrm{P}=0.021)$, age over 21 years $(\mathrm{OR}=0.56, \mathrm{P}<0.001)$, the formation of the schema of recognition $(\mathrm{OR}=0.54, \mathrm{P}=0.01)$ and the formation of the schema entitlement $(\mathrm{OR}=0.47, \mathrm{P}=0.002)$ with the reduction and the formation of the schema of social isolation / emotional inhibition ( $\mathrm{OR}=2.94, \mathrm{P}=0.006)$ was accompanied with increasing of the chance of being addicted to social networks.

Conclusion The results showed that some of the individual characteristics and the formation of some of the schemas are associated with decreasing and increasing the chance of addiction to social networks.

\section{Extended Abstract}

\section{Introduction}

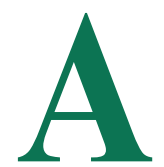

ccess to the Internet is increasingly easy due to advances in mobile technology and the prevalence of smartphones [1,2]. Social Network Sites (SNSs) are virtual communities where users can create individual public profiles, interact with real-life friends, and meet other people based on shared interests [3].

Compared to the general population, teenagers and students are the most frequent users of SNSs [4]. Kuss \& Griffiths also found that between $55 \%$ and $82 \%$ of teenagers and young adults use SNSs regularly [5]. Kim and et al.'s study has shown that seeking friends, social support, information, and entertainment were found to be the most

* Corresponding Author:

Masoumeh Alimohammadi

Address: Department of Psychology, Shahrood Branch, Islamic Azad University, Shahrood, Iran.

Tel: +98 (912) 3321662

E-mail: alimohammadi.m1@gmail.com 
significant motivations for SNSs usage in a sample of 589 undergraduate students [6].

Addiction to SNSs is an international issue with numerous Methods of measurement [7]. The transition from normal to problematic SNSs use occurs when SNS is viewed by the individual as an important (or even exclusive) mechanism to relieve stress, loneliness, or depression [8].

Tang \& Koh's study showed that $29.5 \%$ of Singaporean college students possess SNS addiction [10]. Early maladaptive schema evolves in early childhood through negative experiences with key characters in one's life. Maladaptive schemas are the cognitive infrastructures that lead to the formation of irrational beliefs. Early maladaptive schema has a profound effect on thought, feeling, behaviors, and the way people interact with others [14]. Maladaptive schemas are not directly responsible for personality disorders, although, they increase one's vulnerability toward disorders [15]. Therefore, a hypothesis was formulated: there is a significant relationship between the early maladaptive schema and SNSs addiction.
Given the concerns outlined above, the present study attempted to find an academic answer to the above hypothesis. Such knowledge will also inform more effective intervention of problematic use of SNSs, and prevention of the development of addiction to SNSs among medical and allied health students.

\section{Methods}

This cross-sectional study was performed on medical and allied medical science students of Semnan University of Medical Sciences in 2019. Sampling was done by stratified random. The sample size was 384 people based on the Cochran formula. In this study, Yang Schema Questionnaire and Mobile-based Social Network Addiction Questionnaire were used as data collection tools.

\section{Results}

A total of 215 students (61.4\%) had at least one early maladaptive schema, while 135 (38.6\%) had no maladaptive schema. The mean score of early maladaptive schemas for students was $188.22 \pm 41.66$ and $45(12.9 \%)$ of them had a

Table 1. Association between early maladaptive schemas items and addictive initiatives to mobile social networks in terms of crude and adjusted odds ratio (OR)

\begin{tabular}{|c|c|c|c|c|c|c|c|c|c|c|}
\hline \multirow{3}{*}{ Variables } & \multirow{2}{*}{\multicolumn{2}{|c|}{ No. (\%) }} & \multicolumn{2}{|c|}{ Simple Model } & \multicolumn{2}{|c|}{ Multiple Model } & \multicolumn{4}{|c|}{ Reduced Model } \\
\hline & & & \multirow{2}{*}{ Crude OR } & \multirow[b]{2}{*}{$\mathbf{P}$} & \multirow{2}{*}{$\begin{array}{l}\text { Adjusted } \\
\text { OR }\end{array}$} & \multirow[b]{2}{*}{$\mathbf{P}$} & \multirow{2}{*}{$\begin{array}{l}\text { Adjusted } \\
\text { OR }\end{array}$} & \multicolumn{2}{|c|}{$95 \% \mathrm{Cl}$} & \multirow[b]{2}{*}{$\mathbf{P}$} \\
\hline & $\begin{array}{l}\text { Healthy } \\
\text { Users }\end{array}$ & $\begin{array}{l}\text { Addicted } \\
\text { Users }\end{array}$ & & & & & & Lower & Upper & \\
\hline Sex (Female) & $124(75.6)$ & 95 (51.1) & 0.33 & $<0.001$ & 0.37 & $<0.001$ & 0.35 & 0.215 & 0.58 & $<0.001$ \\
\hline Age (>21) & $64(40.2)$ & $51(27.4)$ & 0.56 & 0.016 & 0.55 & 0.019 & 0.56 & 0.35 & 0.91 & 0.021 \\
\hline Failure & $1(0.6)$ & $4(2.2)$ & 2.14 & 0.022 & 1.43 & 0.336 & - & - & - & - \\
\hline Social Isolation & $2(1.2)$ & 994.8) & 2.24 & 0.026 & 2.88 & 0.011 & 2.94 & 1.36 & 6.37 & 0.006 \\
\hline $\begin{array}{c}\text { Emotional depriva- } \\
\text { tion }\end{array}$ & $15(9.1)$ & 31 (16.7) & 1.22 & 0.454 & - & - & - & - & - & - \\
\hline Defectiveness & $8(4.9)$ & $11(5.9)$ & 1.60 & 0.036 & 1.12 & 0.671 & - & - & - & - \\
\hline Draw attention & $34(20.7)$ & 25 (13.4) & 0.55 & 0.007 & 0.54 & 0.014 & 0.54 & 0.33 & 0.86 & 0.010 \\
\hline Clutter & $9(5.5)$ & $16(8.6)$ & 1.17 & 0.565 & - & - & - & - & - & - \\
\hline Detachment & $11(6.7)$ & $16(8.6)$ & 1.45 & 0.179 & 0.99 & 0.999 & - & - & - & - \\
\hline Vulnerability to harm & $9(5.5)$ & $16(8.6)$ & 0.76 & 0.330 & - & - & - & - & - & - \\
\hline Self-sacrifice & $7(4.3)$ & $11(5.9)$ & 1.90 & 0.024 & 1.64 & 0.144 & 1.68 & 0.90 & 3.16 & 0.102 \\
\hline $\begin{array}{l}\text { Punishment / unfair } \\
\text { standards }\end{array}$ & $37(22.6)$ & $28(15.1)$ & 0.067 & 0.080 & 0.68 & 0.147 & - & - & - & - \\
\hline Merit & $51(31.1)$ & 33 (17.7) & 0.48 & 0.001 & 0.52 & 0.009 & 0.47 & 0.29 & 0.76 & 0.002 \\
\hline Mistrust & 34 (20.7) & 36 (19.4) & 1.007 & 0.975 & - & - & - & - & - & - \\
\hline
\end{tabular}


score higher than average. The mean (or median) score for all domains (except the guided by others/attention) in students with addiction to social networks was higher so that the difference in the scheme of detachment and exclusion $(\mathrm{P}=0.004)$, guided by others $(\mathrm{P}=0.024)$, and self-regulation and impaired performance $(\mathrm{P}=0.002)$ were significant. The female gender $(\mathrm{OR}=0.35, \mathrm{P}=0.021)$, age over 21 years $(\mathrm{OR}=0.56, \mathrm{P}<0.001)$, the formation of the schema of recognition $(\mathrm{OR}=0.54, \mathrm{P}=0.01)$, and the formation of the schema entitlement $(\mathrm{OR}=0.47, \mathrm{P}=0.002)$ with the reduction and the formation of the schema of social isolation/emotional inhibition ( $\mathrm{OR}=2.94, \mathrm{P}=0.006)$ was accompanied with increasing of the chance of being addicted to social networks.

\section{Discussion}

The results of this study, on the one hand, showed that social network addiction is a complex phenomenon that is related to psychological, social, and cultural factors; on the other hand, it showed the depth and complexity of early maladaptive schemas in challenges and phenomena among young people, especially students. Given the emergence of new technologies in the future, these results enable us to further root out future trends among young people and develop strategies for intervention programs such as schema therapy to prevent and treat the problems among young people. Thus, educational institutions can guide the use of social networks among students to improve education.

\section{Ethical Considerations}

Compliance with ethical guidelines

The study approved by Ethics Committee of Semnan University of Medical Sciences (IR.SEMUMS. REC.1396.140).

\section{Funding}

The research was supported by Semnan University of Medical Sciences (Grant No.: 1457).

\section{Authors contributions}

Study design: Mehdi Kahouei, Masoumeh Alimohammadi; Acquisition of data: Fatemeh Paknazar, Ghazaleh Mosayebi; Data analysis: Fatemeh Paknazar; Interpreting the results: Mehdi Kahouei, Masoumeh Alimohammadi; Writing - review \& editing, approve the final version of the manuscript: All authors.

\section{Conflicts of interest}

The authors declared no conflict of interests.

\section{Acknowledgements}

We would like to thank the Clinical Research Development Unit of Kowsar and Amir-al-Momenin Educational, Research and Therapeutic Centers of Semnan University of Medical Sciences for providing facilities to this work. 


\title{
ارتباط بين طرحوارههاى ناساز كار اوليه با اعتياد به شبكههاى اجتماعى در بين دانشجويان دانشكاه علوميزشكى سمنان
}

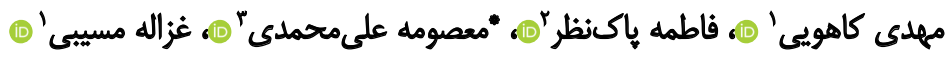 \\ 1. أشككده بيرايزشكى، مركز تحقيقات عوامل اجتماعى موثر بر سلامت ، دانشكاه علوميزشكى سمنان، سمثنان، ايران.

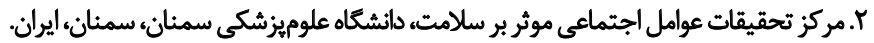

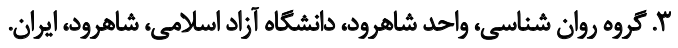

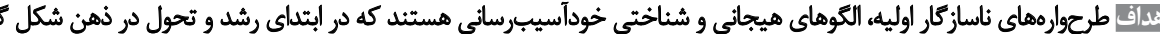

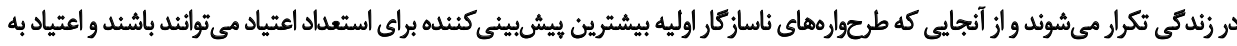

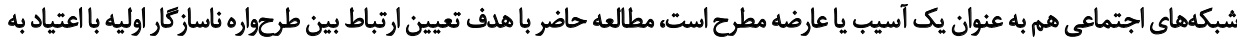

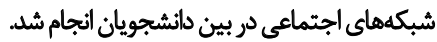

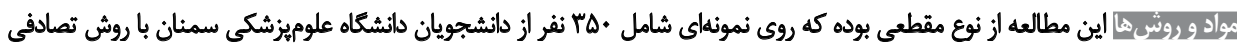

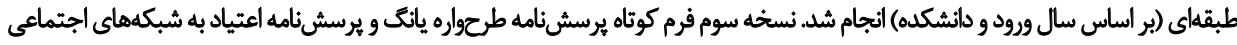

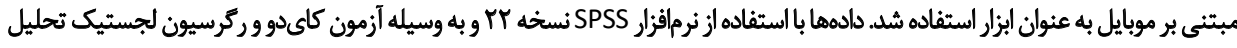

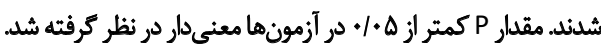

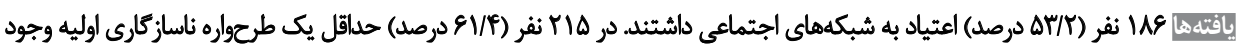

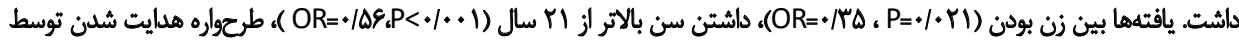

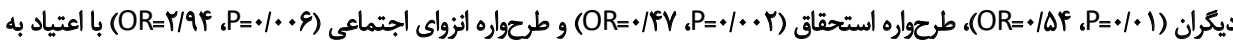

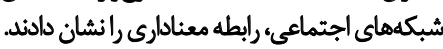

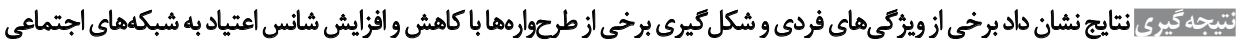
همراهات.

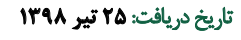

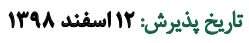
تاريخ انتشار: Vi تير

كليدواثهها:

طرحواره ناسازكًار اوليه.

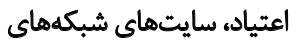
اجتماعى، دانشجويان

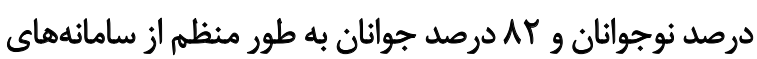

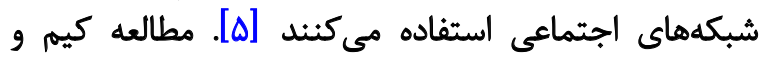

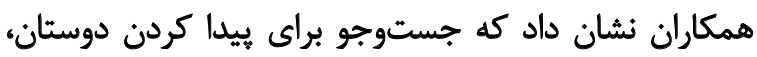

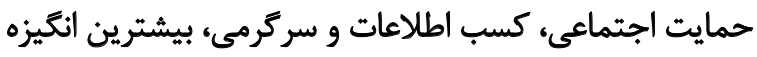

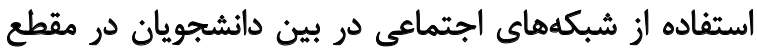
كارشناسى بوده است [عاد إن.

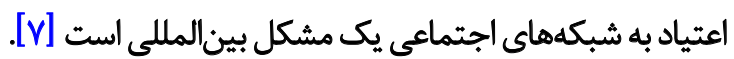

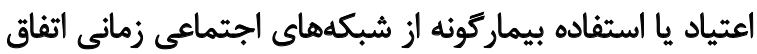

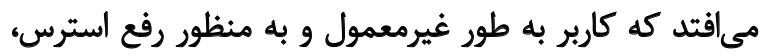

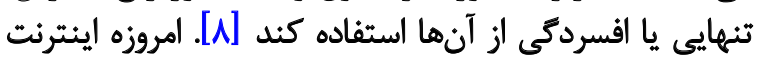

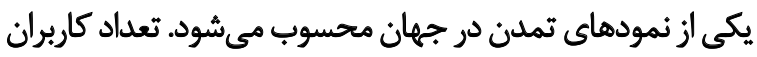

مقدمه

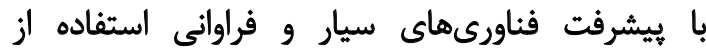

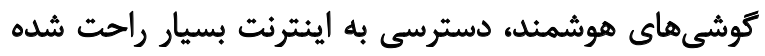

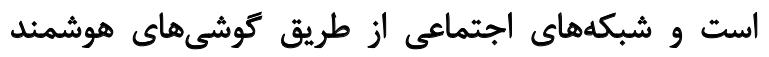

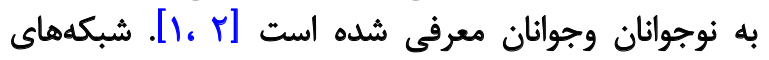

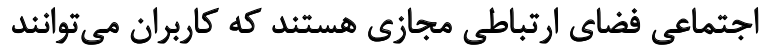

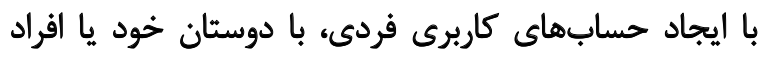

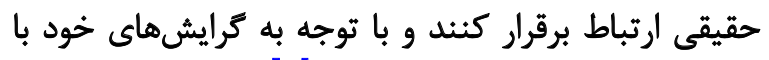

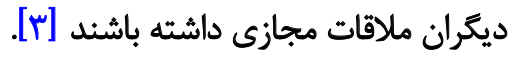
جوانان و دانشجويان بيشترين كاربران شبكههاى اجتماعى

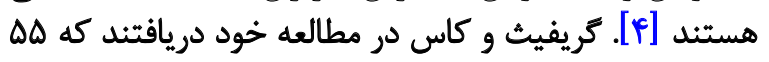
تلفن: alimohammadi.m1@gmail.com : بـث الكترونيكي: 
عوارض ناشى از آن ميتواند با اختلالات شخصيتى در ارتباط باشد.

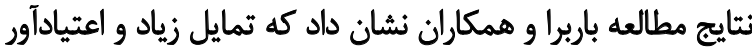

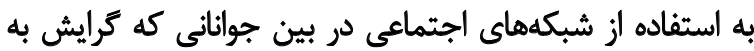

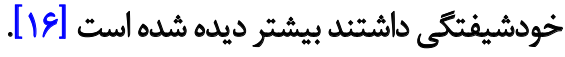

همجنين مطالعه شايق و همكاران نشان داد كه اعتياد به اينترنت

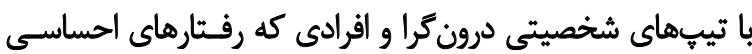

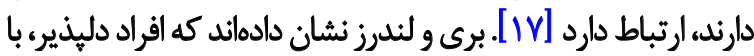

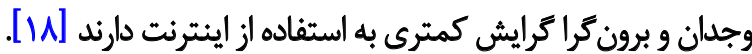

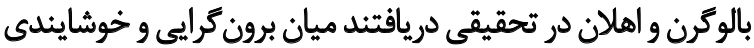

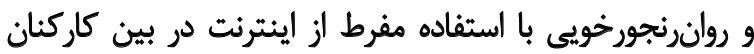
ارتباط معنى دارى وجود دارد [19].

مطالعات انجامشده در ايران بيشتر به اعتياد به اينترنت كه از

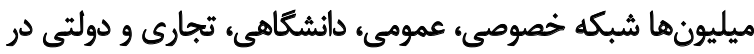

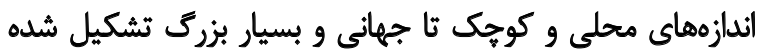

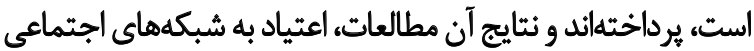

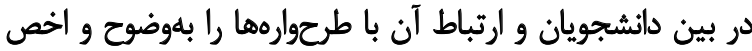

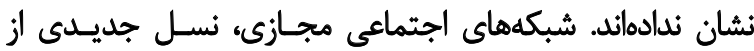

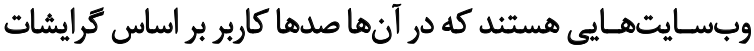

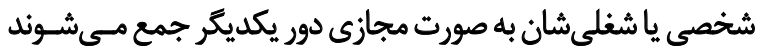

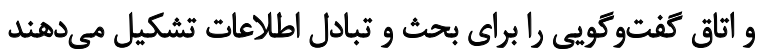

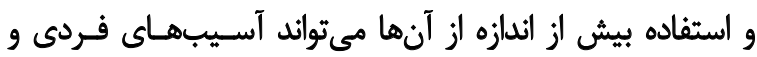

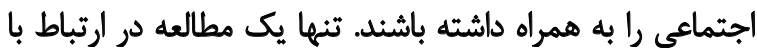

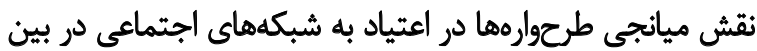

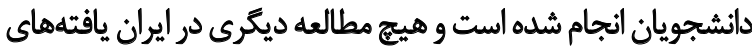

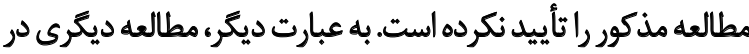

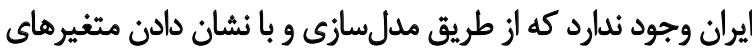

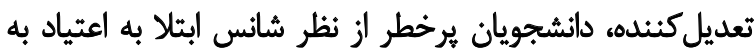

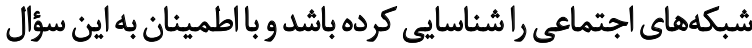

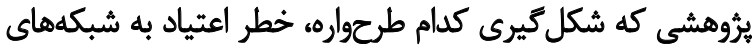

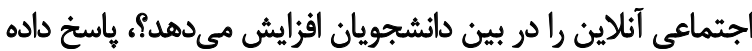

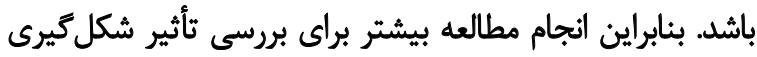

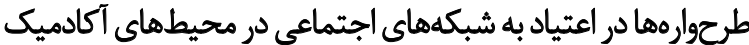

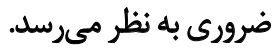

با توجه به خلأيروهشى فوق و وجود ارتباط طرحوارههاى ناساز آرار

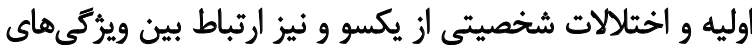

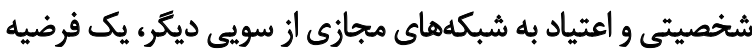

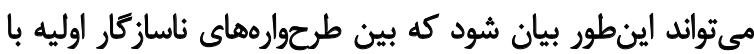

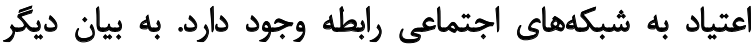

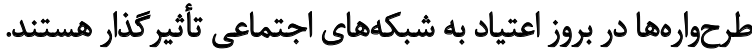

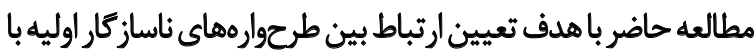

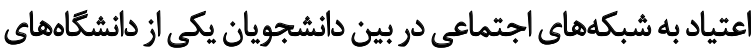

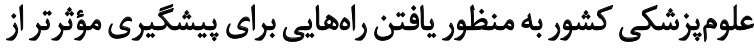
اين نوع اعتياد در بين دانشجويان كروه يزشكى انجام شد.

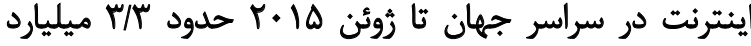

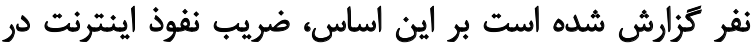

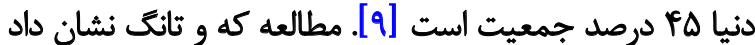

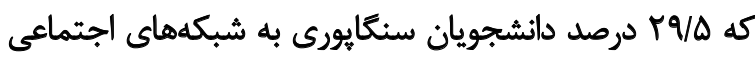

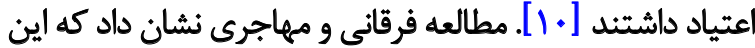

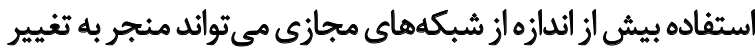

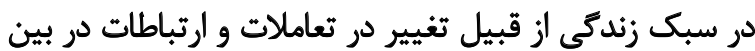

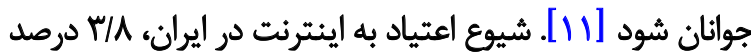

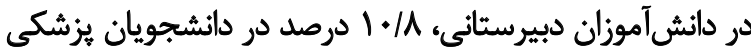

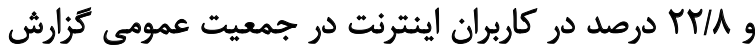

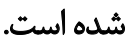

در سايت آمار جهانى اينترنت، ضريب نفوذ اينترنت در ايران

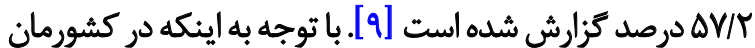

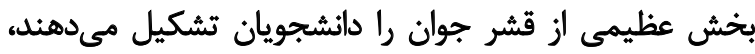

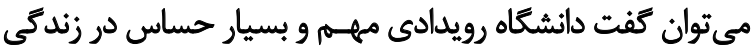

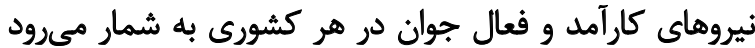

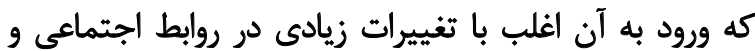

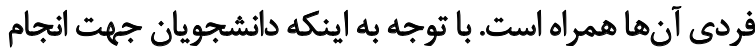

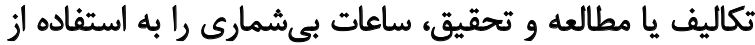

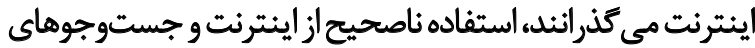

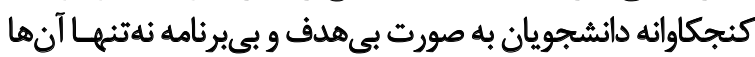

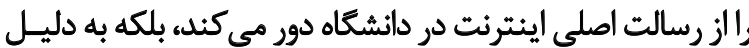

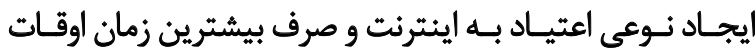

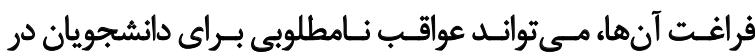

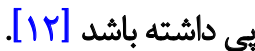
با توجه به اينكه دانشجويان شاغل به تحصيل در رشته

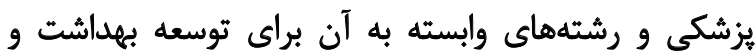

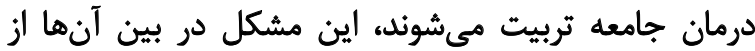

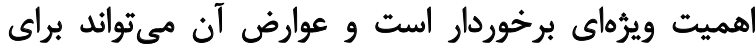
جامعه تبعات نامطلوبي به همراه داشته باشد [اش آن]

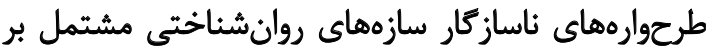

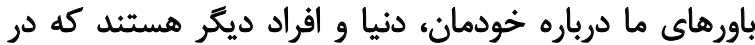

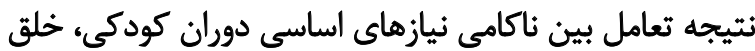

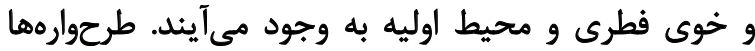

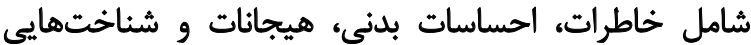

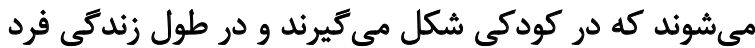

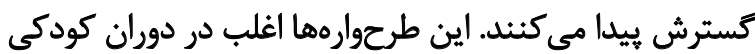

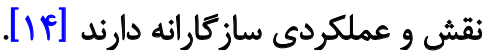

طرحوارههاي ناساز كار اوليه مسئول مستقيم بيدايش اختلالات

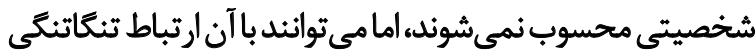

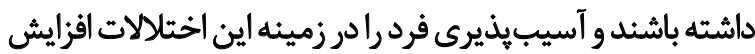

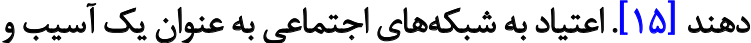




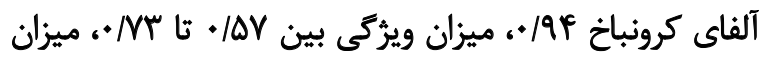

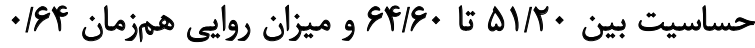

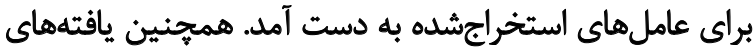

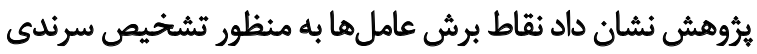

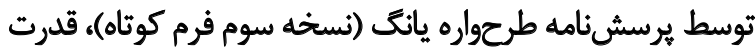

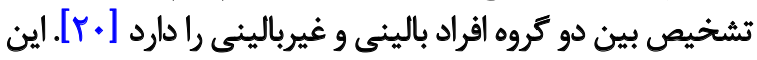

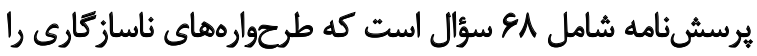

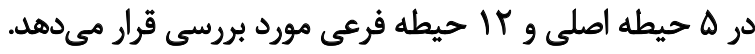

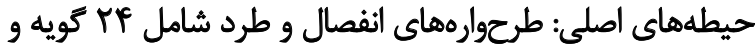

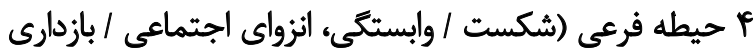

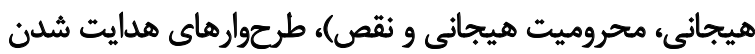

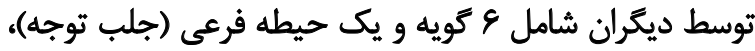

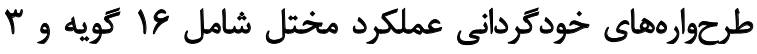

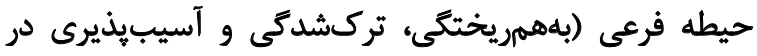

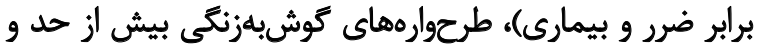

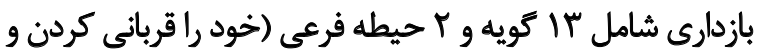

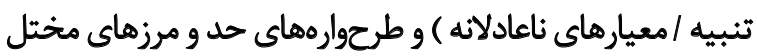

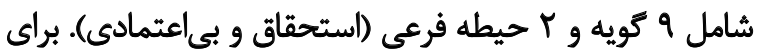

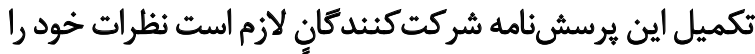

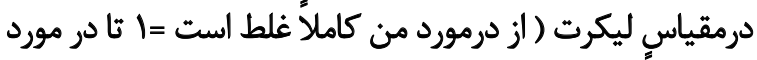

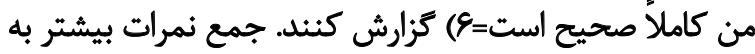

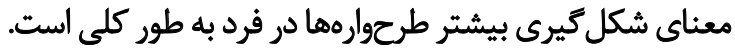

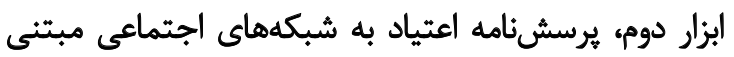

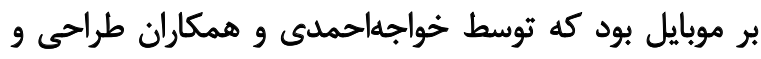

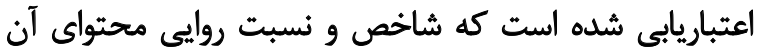

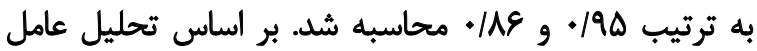

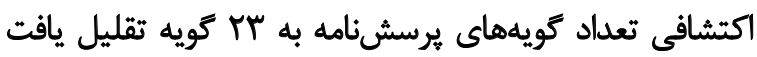

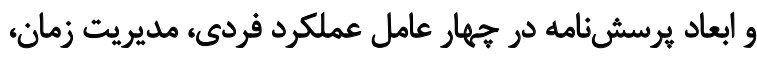

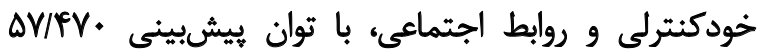

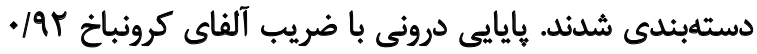

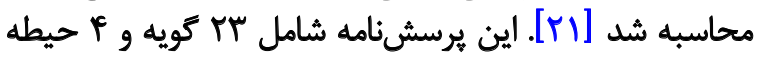

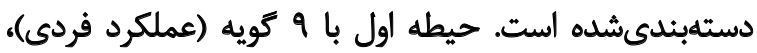

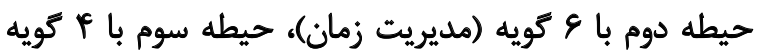

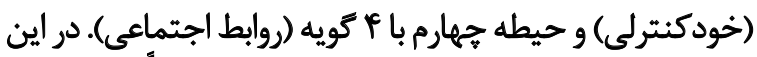

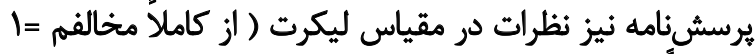

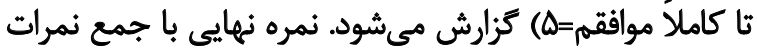

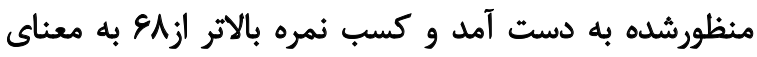

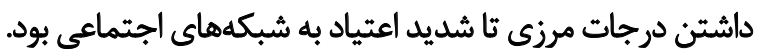

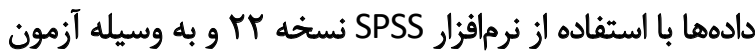

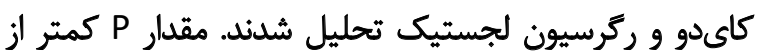

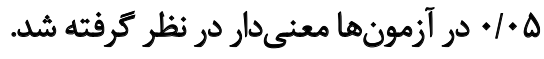

يافتهها

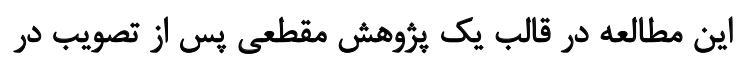

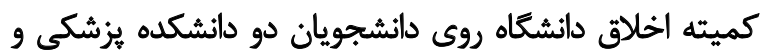

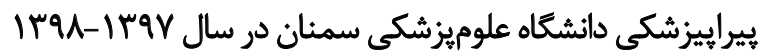

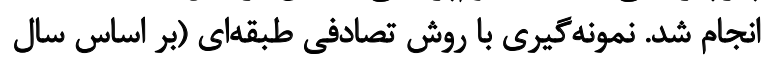

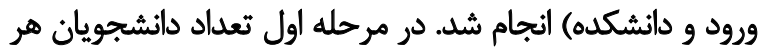

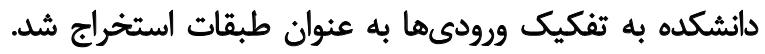

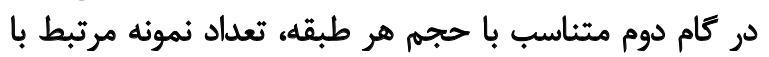

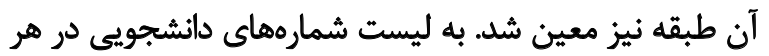

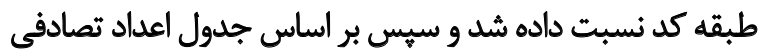

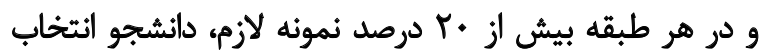

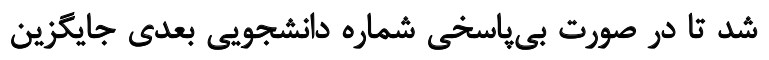

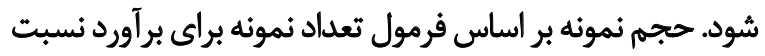

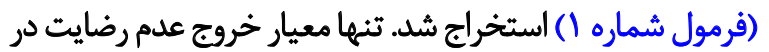

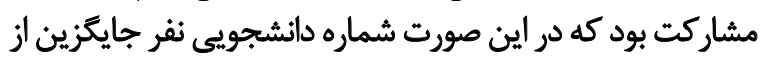
قبل تعيين شده بود.

$$
\begin{aligned}
& p=\frac{1}{2}, q=\frac{1}{2}, z=1.96, d=0.05 \\
& n=\frac{z^{2} p q}{d^{2}}=\frac{1.96^{2} \times \frac{1}{2} \times \frac{1}{2}}{0.05^{2}} \approx 384
\end{aligned}
$$

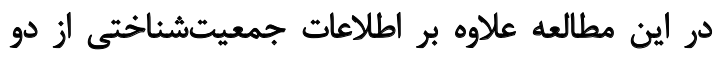

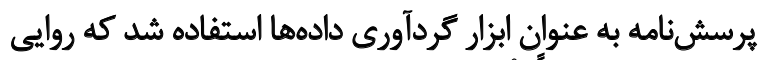

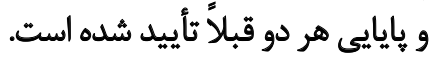

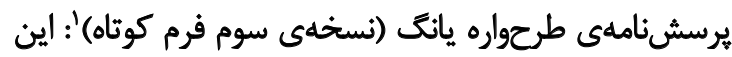

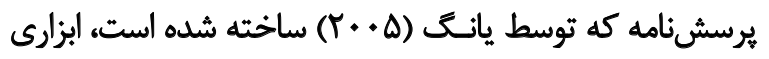

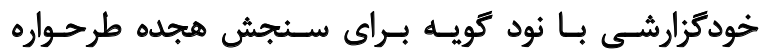

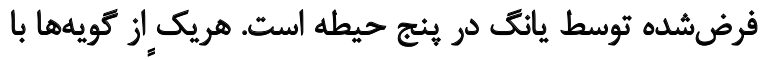

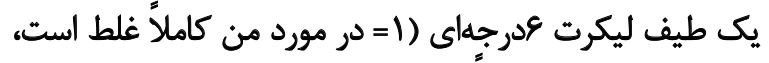

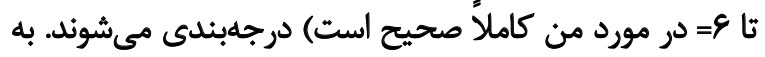

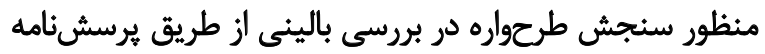

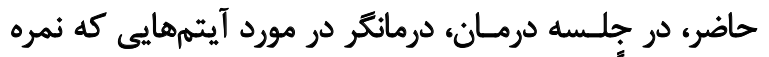

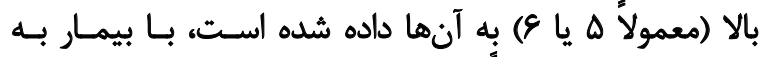

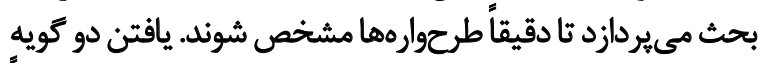

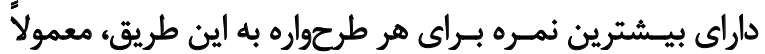

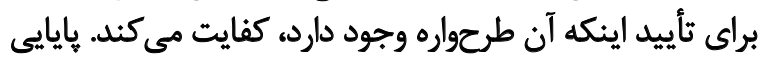

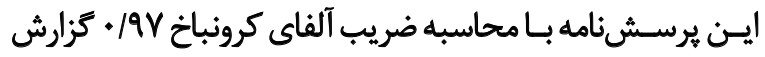

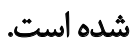

ابزار نخست مورد استفاده در مطالعه، نسخه سوم فرم كوثاه

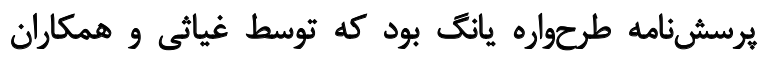
به زبان فارسى بركردان و روانسنجى شده است كه ضريب

1. Young Schema Questionnaire 
جدول ا. توزيع فراوانى دانشجويان شركت كنيده در مطالعه از نظر ويزّكى هاى فردى

\begin{tabular}{|c|c|c|}
\hline تعداد (درصد) & $\log$ & ويؤكى هاى فردى \\
\hline$|r|(r V / F)$ & مرد ل & \multirow{3}{*}{ جنسيت } \\
\hline & & \\
\hline$r 19(\& F / 8)$ & is & \\
\hline $\ln \left(m / m^{\circ}\right)$ & بالاى آ سال & \multirow[b]{2}{*}{ سن س } \\
\hline$M \pi(8 / 8)$ & ال سال و هايينتر & \\
\hline MIO $(8 / / P)$ & كارشناسى & \multirow[b]{2}{*}{ مقطع تحصيلى } \\
\hline Iro (rNe) & دكتراي حرفهاي & \\
\hline$W \in(\Delta r / T)$ & بله & \multirow{2}{*}{ اعتياد به شبكههاى اجتماعى } \\
\hline $\operatorname{Ixt}(\kappa \& / A)$ & خير & \\
\hline $\operatorname{MIO}(8 / / 4)$ & بله & \multirow{3}{*}{ شكل كيرى حداقل يك طرحواره ناساز كارى اوليه } \\
\hline & & \\
\hline$I \Psi \Delta(T N \mathcal{N})$ & خير & \\
\hline
\end{tabular}

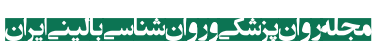

شبكههاى اجتماعى در جدول شماره ب نشان داده شده است.

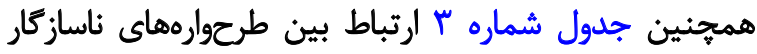

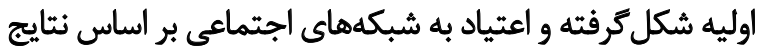

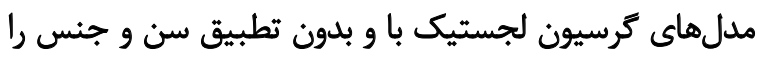

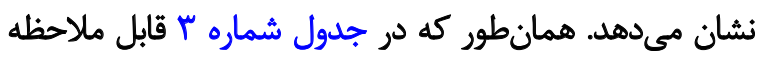

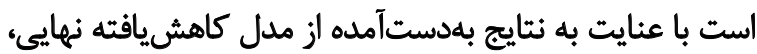

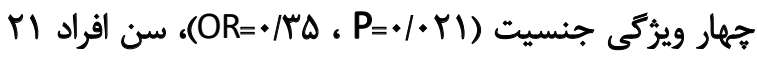

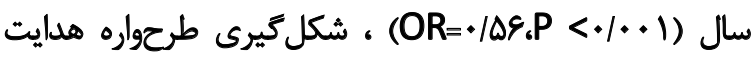

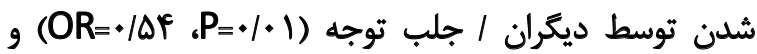

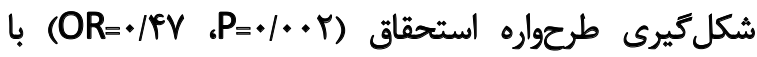

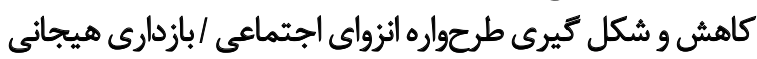

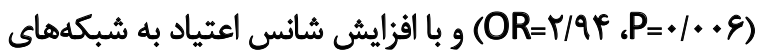

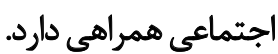

بحث

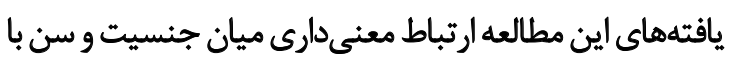

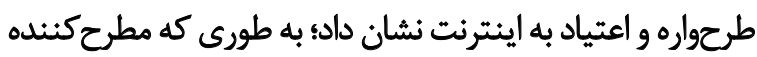

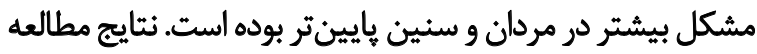

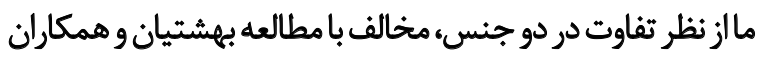

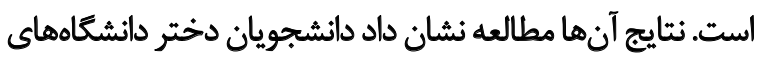

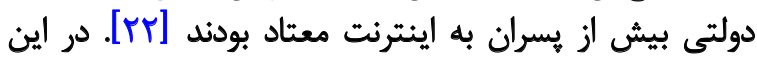

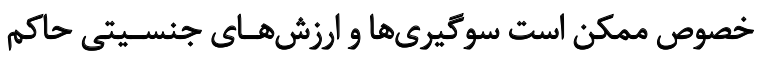

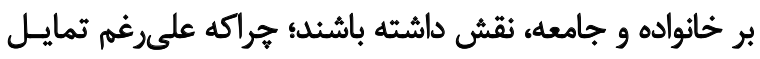

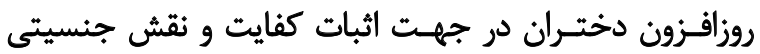

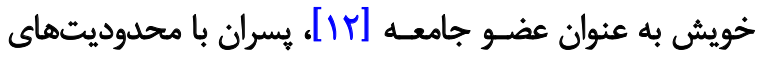

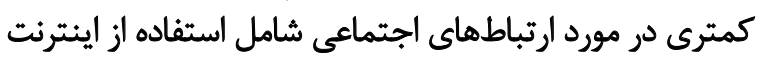

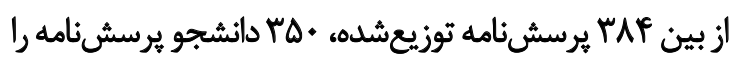

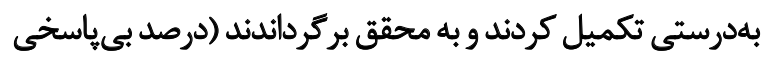

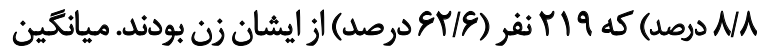

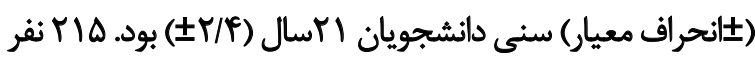

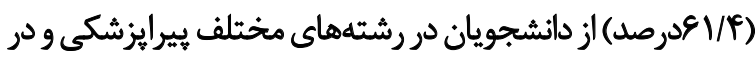

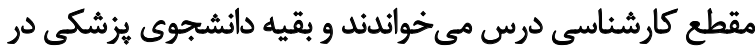

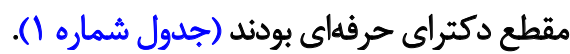

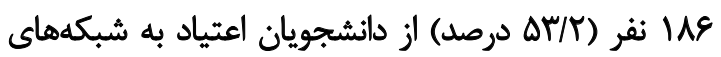

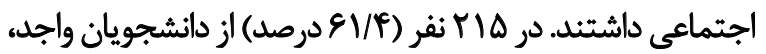

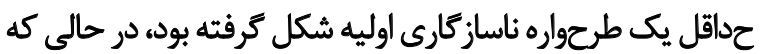

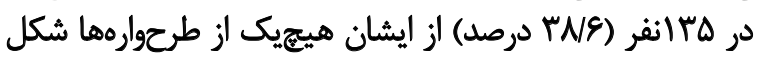

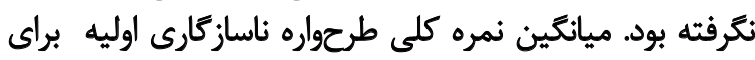

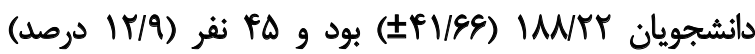

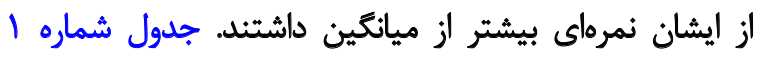

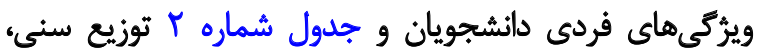

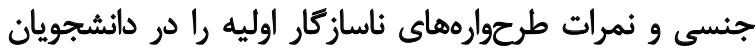

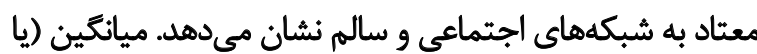

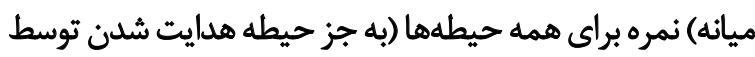

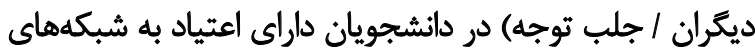

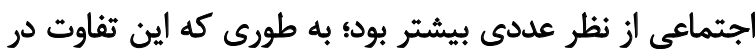

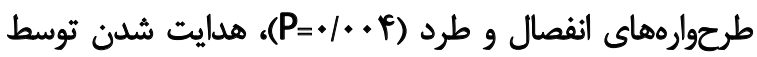

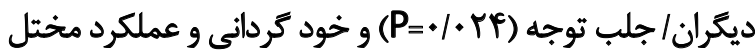

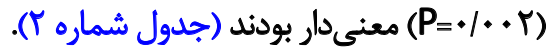

توزيع فراوانى شكلىيرى طرحوارهها به تفكيك هريك

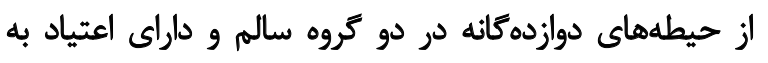


جدول r. توزيع سنى ، جنسى و نمرات طرحوارهاي ناسازكًار اوليه بين دانشجويان معتاد به شبكههاى اجتماعى و سالم

\begin{tabular}{|c|c|c|c|c|c|}
\hline \multicolumn{2}{|c|}{ آماره } & \multicolumn{3}{|c|}{ تعداد و مياتكين||نحراق معيار يا ميانه (دامنه ميان ثاركى) } & \multirow{2}{*}{ ويؤيى } \\
\hline مقدار بيى & $z \ln x^{r} \ln t$ & كاربران معتاد (1) (نفر) & كاربران سالم(ع) (نفر) & تعداد كل (+مانقر) & \\
\hline$<\bullet / \cdot+1$ & . TMH & 90,91 & inf, t. & riq, $|r|$ & جنس (مردو زن) \\
\hline $.1 \cdot 1 \mathrm{~A}$ & tree & $r \cdot(r \cdot, M)$ & $M(Y \cdot g r)$ & $M(T \cdot g r)$ & ن \\
\hline .1 .81 & $V / M$ & IQT/IY \pm YNTA & $M r / M^{ \pm} \pm r f / a r$ & WWTEE PVIES & نمرات طرحوارهها \\
\hline 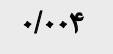 & r/q. & $\Delta q(p q, V r)$ & $\Delta T / \Delta(P \Delta, g N / N \Delta)$ & $\Delta V(P V, E Q / T \Delta)$ & انفصال و طرد \\
\hline$\cdot M \cdot r$ & $1 / 8 x$ & $r \in(r i, M)$ & $r \Delta(r+R \Delta, r \cdot)$ & $r \in(T, g T)$ & شكست / وابستكى \\
\hline $.11 \cdot 8$ & V/EIV & $10(11 / N 0,11)$ & If $(1 \cdot g$ IV) & If $(11, \mid 1)$ & انزواى اجتماعى / بازدارى هيجانى \\
\hline$\%$ & r/qTo & $\|(N, \mid f)$ & $\lambda(*, \| r)$ & $1 \cdot(\varepsilon, \mid f)$ & محروميت هيجانى \\
\hline$\%$ & $\% / .90$ & $v(\Delta, 1 \cdot)$ & $8(f, 9)$ & $g(f, g) \cdot 1$ & نقص \\
\hline$\cdot \%$ & T/TAY & $19(10, \pi)$ & $M(M, G E)$ & $T(1 \Delta, T \Delta)$ & هليت شلن توسط ديكران / جلب توجه \\
\hline$\%+r$ & $r /+8 \Delta$ & $M F(M F, \Delta H)$ & $r q(T A, \Delta \backslash / Y A)$ & $H T(T+g \Delta T / T \Delta)$ & خودكّردانى و عملكرد مختل \\
\hline .10 .1 & T/TEA & $19(17, M i)$ & $1 \Delta(1 . / T \Delta, T Y / V \Delta)$ & IV $\left(I T, T T^{\prime}\right)$ & به بهن \\
\hline $.1 \cdots \infty$ & thaf & $N \Delta(8,11)$ & $V(f, 1 \cdot)$ & $\left.V\left(\Delta_{g}\right) I\right)$ & تركشدكى \\
\hline.$/ N a r$ & I/RTr & $10(19,19)$ & If $(q, r \cdot N \Delta)$ & $10\left(11, T^{\prime} \cdot\right)$ & آسيبذيذيرى در برابر ضرر و بيمارى \\
\hline.$M E$ & I/MTE & $r q r+ \pm 1 \cdot 18 r$ & PVIRT $\pm \|$ W/AP & rNFE $\pm 11 / \cdot V$ & كوشبهزئكى بيش از حلد و بازدارى \\
\hline$+1++1$ & r/TrA & $M(I V, Y A)$ & $19(1 f, r e / V \Delta)$ & $M(1 \Delta, T Y)$ & خود را قربانى كردن \\
\hline. $\mid+\varphi 1$ & $r / \cdot \mu$ & $18(17,19)$ & $I V(I r / r \Delta, r)$ & IV $(I r, g+)$ & تنبيه / معيارهاى ناعادلانه \\
\hline 1.94 & I/NAV & TNES \pm V/RT & $r \cdot / r \cdot \pm N(\Delta)$ & YQ/RUE $\pm N M T$ & حل و مرزهاى مختل \\
\hline$+1+r$ & $r / .94$ & $18(1 r, r+)$ & $M(I F, M)$ & IV $(\mid r, g)$ & استحقاق \\
\hline $.18 \pi T$ &.$/$ fr & $\pi(1 \cdot, 10)$ & $\operatorname{Ir}(9,10)$ & $I r(1 \cdot, \mid \theta)$ & بى اعتمادى \\
\hline
\end{tabular}

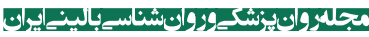

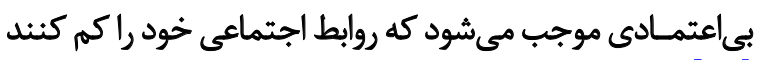

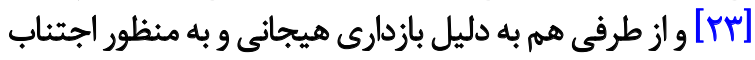

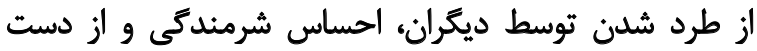

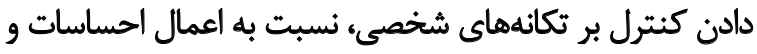

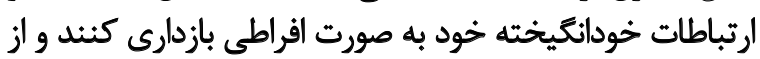

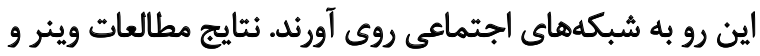

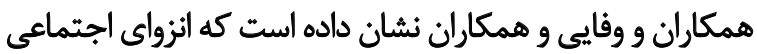

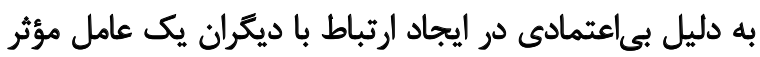

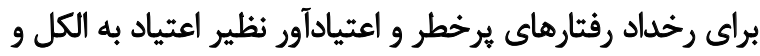

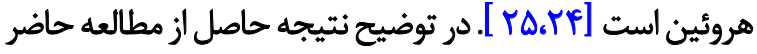

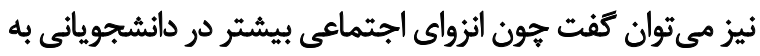

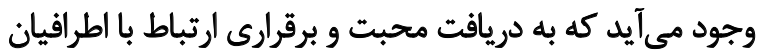

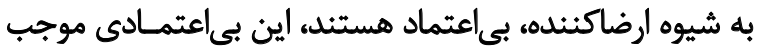

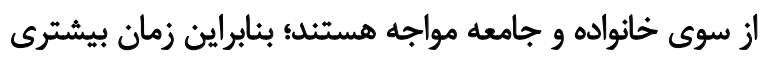

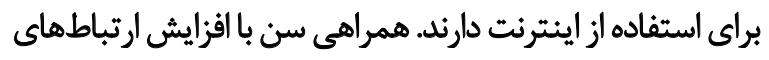

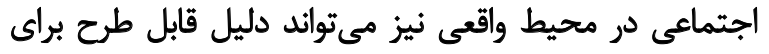
بيشتر بودن مشكل در سنين يايينتر باشد.

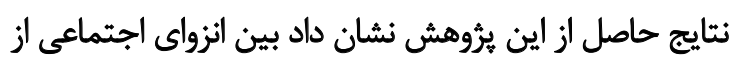

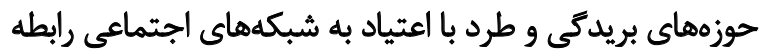

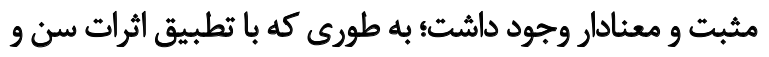

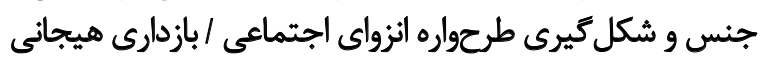

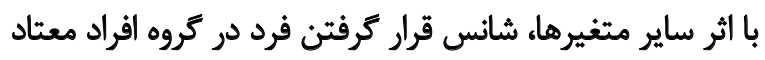

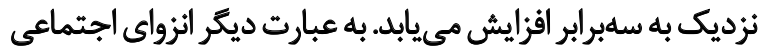

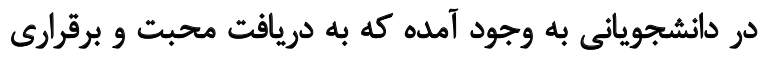

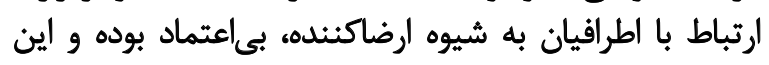


جدول r. ارتباط بين شكل كيرى طرحوارههاى ناسازكار اوليه و اعتياد به شبكههاى اجتماعى بر اساس مدلهاى ركرسيون لجستيك

\begin{tabular}{|c|c|c|c|c|c|c|c|c|}
\hline \multicolumn{6}{|c|}{ مدل نههايي } & \multicolumn{2}{|c|}{ تعداد (درصد) } & \multirow{3}{*}{ ويؤَى } \\
\hline \multirow[t]{2}{*}{$\mathbf{P}$} & \multicolumn{2}{|c|}{ فاصله اطمينان 90} & \multirow{2}{*}{ تسبت شانيق } & \multirow{2}{*}{ ضريب } & \multirow{2}{*}{ والد } & \multirow{2}{*}{ 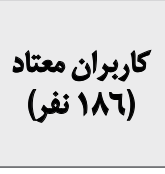 } & \multirow{2}{*}{ كاربران سالم } & \\
\hline & حد بإيين & حد بالا & & & & & & \\
\hline \multirow{2}{*}{$<+1 \cdot+1$} & \multirow{2}{*}{$\cdot / r)$} & \multirow{2}{*}{$\cdot 101$} & $\cdot / r \omega$ & \multirow{2}{*}{$-1 /+\infty$} & \multirow{2}{*}{ Ir/Te } & $q \Delta(\Delta) / 1)$ & ITf $(\mathrm{NO/F})$ & \multirow[b]{2}{*}{ جنسيت } \\
\hline & & & 1 & & & 9) (PNQ) & $r+(\pi r / r)$ & \\
\hline \multirow{2}{*}{$\cdot \mid \cdot r)$} & \multirow{2}{*}{.$/ N \Delta$} & \multirow{2}{*}{.$/ 91$} &.$/ \Delta F$ & \multirow{2}{*}{$-\cdot / \Delta V$} & \multirow{2}{*}{$\Delta / m$} & D) $(T V / F)$ & $e \in(f \circ / T)$ & بالاى آ سال \\
\hline & & & 1 & & & 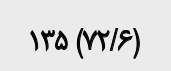 & $M(\Delta N A)$ & ا إ سال و كمتر \\
\hline- & - & - & - & - & - & $F(T / M)$ & $1(\cdot / 9)$ & شكست / وابستكى \\
\hline $.1+.9$ & $1 / 48$ & $s / \pi$ & r/qF & $V / v$ & g/Ke & $9(F / A)$ & $r(M / T)$ & انزواى الجتماعى / بازدارى هيجانى \\
\hline- & - & - & - & - & - & $M(I \& M)$ & $10(9 / 1)$ & هحروميت هيجاني \\
\hline- & - & - & - & - & - & $11(\Delta / 9)$ & $\Lambda(f / 9)$ & نقص \\
\hline $.1 \cdot 1$ &.$/ M$ & $\cdot \mathbb{N}^{2}$ & $\cdot / \Delta f$ & -.181 & $e / m$ & $r \Delta(\mid r / T)$ & $M(T \cdot M)$ & جلب توجه \\
\hline- & - & - & - & - & - & $\mathbb{1}(\mathrm{NF})$ & $9(\Delta / \Delta)$ & بd بهن \\
\hline- & - & - & - & - & - & $18(N F)$ & $\Pi(g / V)$ & تركشدكى \\
\hline- & - & - & - & - & - & $18(N F)$ & $9(\Delta / \Delta)$ & آسيبيذيرى در برابر ضرر و بيمارى \\
\hline.$M \cdot r$ &.$/ 9$ & $r / / \&$ & $V / \& A$ &.$|\Delta|$ & $m / M q$ & $11(8 / 9)$ & $V(\kappa / \Psi)$ & خ خودقربانى \\
\hline- & - & - & - & - & - & $r A(\mid \Delta / I)$ & $W(T r / g)$ & تنبيه / معيارهاى ناعادلانه \\
\hline 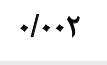 &.$/ 79$ & $\cdot N G$ & $* / \pi Y$ & $-+/ V a$ & g/NF & $\mu(I V / V)$ & $\Delta)(M V /)$ & استحقاث \\
\hline- & - & - & - & - & - & $r g(19 / 4)$ & $m(r+M)$ & بي اعتمادى \\
\hline
\end{tabular}

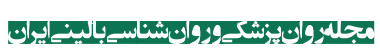

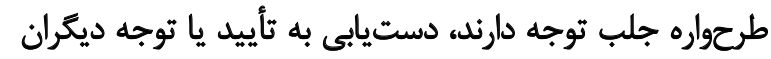

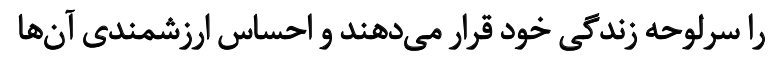

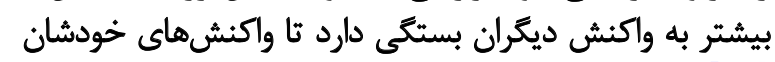

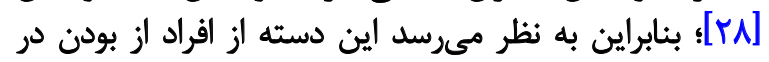

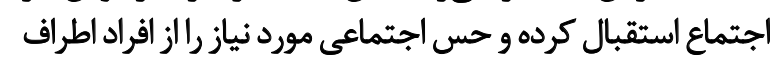

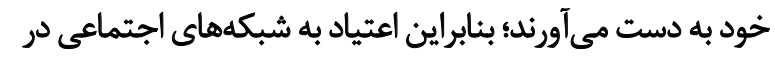
اين افراد كاهش مي ميابد.

شكل كرفتن طرحواره استحقاق با تطبيق ساير اثرات، شانس

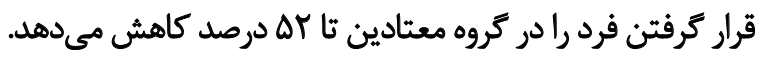

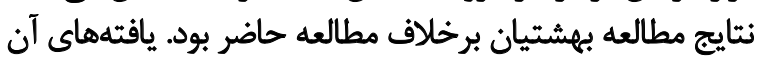

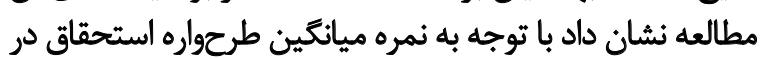

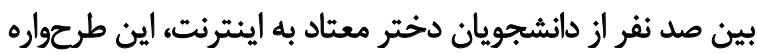

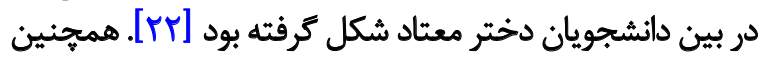

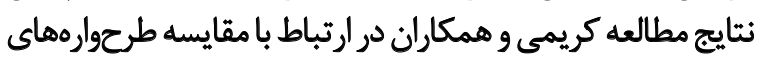

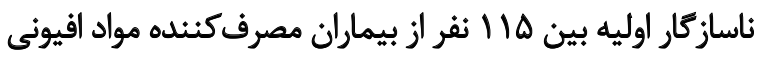

مىشود كه روابط اجتماعى خود را كم كنند و به شبكهاى

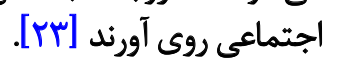

نتايج حاصل از اين يثروهش نشان داد كه طرحواره جلب توجه

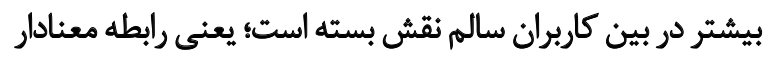

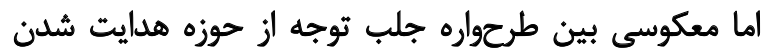

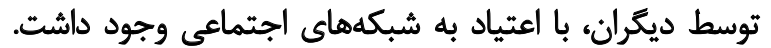

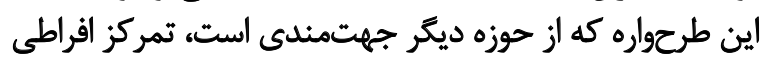

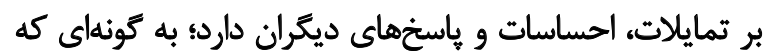

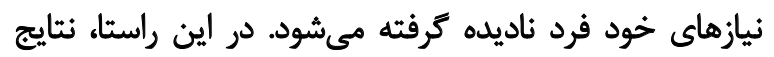

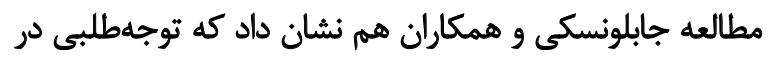

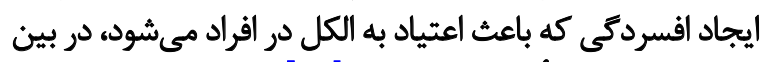

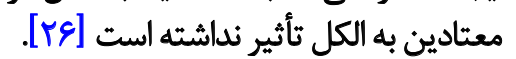

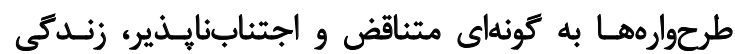

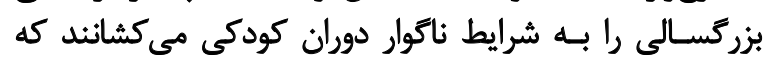

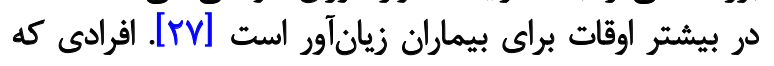


كه مؤسسات آموزشى بثوانند استفاده از شبكههاي اجتماعى در بين دانشجويان رادر جهت بهبود آموزش هدايت كنيند.

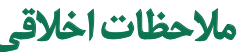

\section{يبيروى أز اصول الخاق تُؤشش}

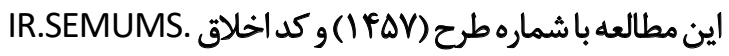

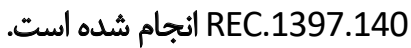

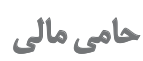

اين مطالعه با حمايت مالى دانشعَاه علوميزشكى سمنان انجام

$$
\text { شده است }
$$

$$
\text { مشاركت ثويسندكان }
$$

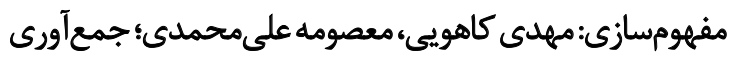

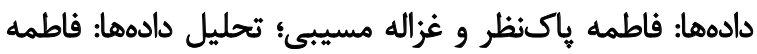

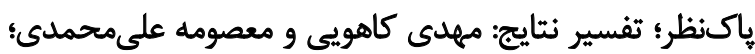

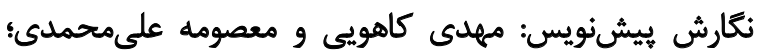
ويراستارى و نهايیىسازى نوشته: همه نويسند كاهن ونان.

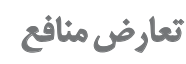

بنابر اظهار نويسندكان اين يُروهش تعارض منافع نداشته است.

$$
\text { تشيكر و قمدرواني }
$$

از كارشناس مركز تحقيقات بالينى بيمارستان كوثر سركار

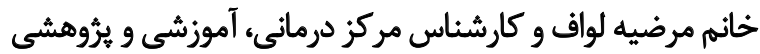

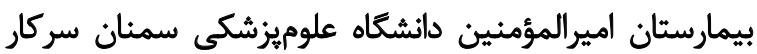

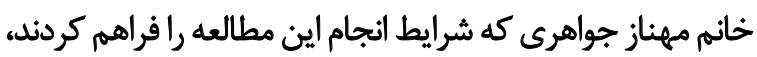

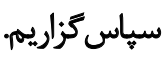

و تحريك كنثله، نشان داد كه طرحواره استحقاق بهمراتب در بين

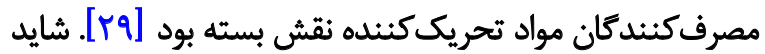

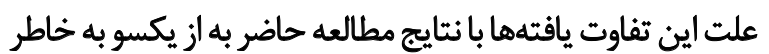

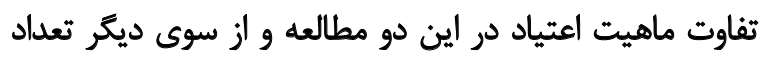

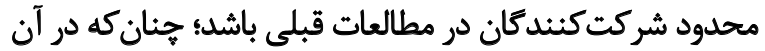

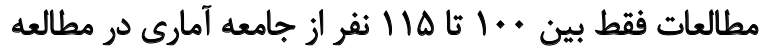

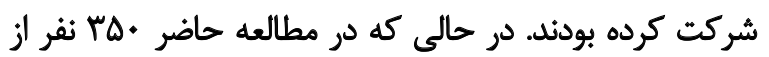
دانشجويان در مطالعه شركت كردند.

با توجه به اينكه افرادى كه داراى طرحواره استحقاق هستند،

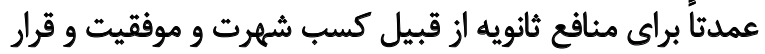

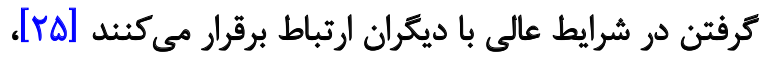

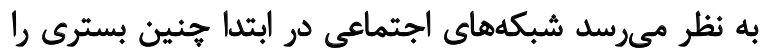

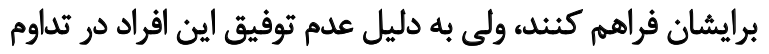

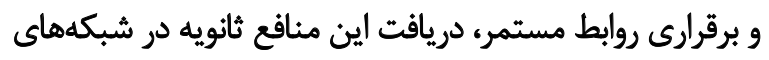

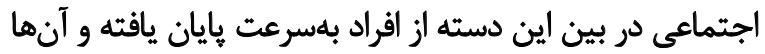

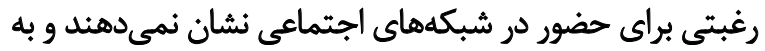

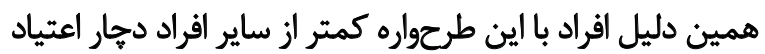
به شبكهاى اجتماعى مى إشوند.

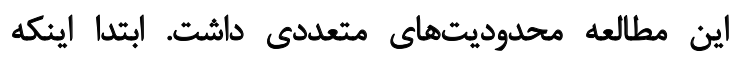

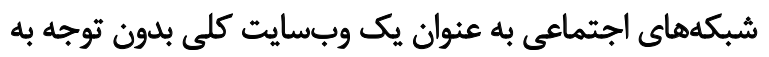

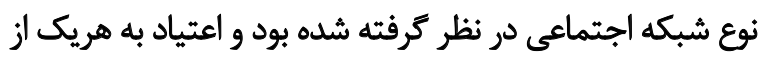

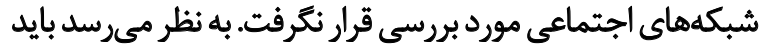

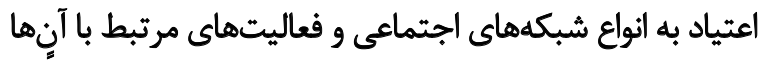

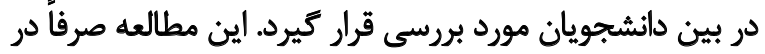

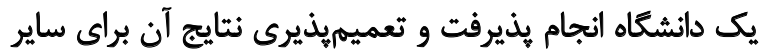

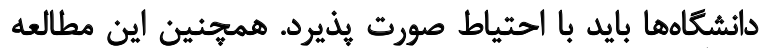

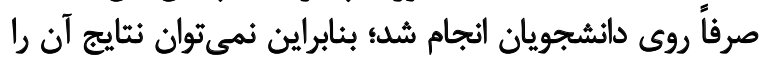

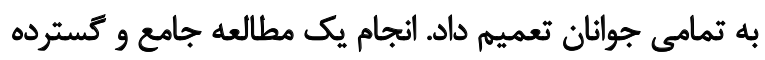

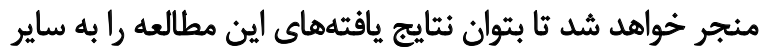

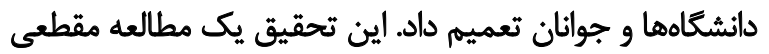

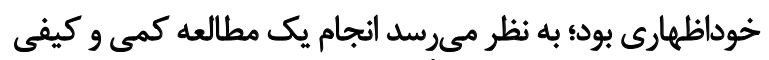

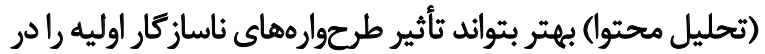
بروز اعتياد به شبكههاى اجتماعى نشان دهد.

\section{نتيجليَّيرى}

نتايج اين مطالعه نشان داد اعتياد به شبكههاى اجتماعى با إنا

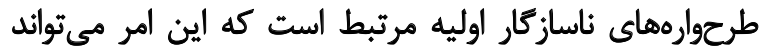

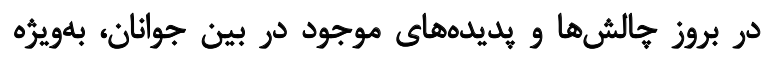

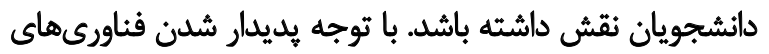

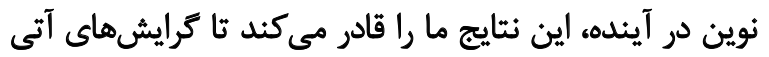

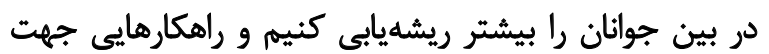

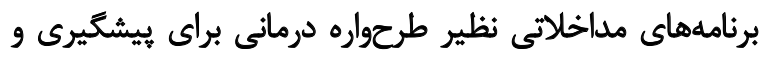

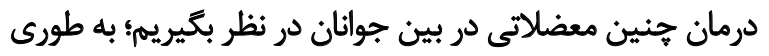




\section{References}

[1] Subrahmanyam K, Reich SM, Waechter N, Espinoza G. Online and offline social networks: Use of social networking sites by emerging adults. Journal of Applied Developmental Psychology. 2008; 29(6):420-33. [DOI:10.1016/j.appdev.2008.07.003]

[2] Farzandipur M, Rangraz Jeddi F, Azimi E. Factors affecting successful implementation of hospital information systems. Acta Informatica Medica. 2016; 24(1):51-5. [DOI:10.5455/aim.2016.24.5155] [PMID] [PMCID]

[3] Barnes SJ, Pressey AD, Scornavacca E. Mobile ubiquity: Understanding the relationship between cognitive absorption, smartphone addiction and social network services. Computers in $\mathrm{Hu}$ man Behavior. 2019; 90:246-58. [DOI:10.1016/j.chb.2018.09.013]

[4] Griffiths MD, Kuss DJ, Demetrovics Z. Social networking addiction: An overview of preliminary findings. In: Rosenberg KP, Feder LC, editors. Behavioral Addictions: Criteria, Evidence, and Treatment. Waltham, MA: Academic Press; 2014. p. 119-141. [DOI:10.1016/B978-0-12-407724-9.00006-9] [PMID]

[5] Kuss DJ, Griffiths MD. Online social networking and addictiona review of the psychological literature. International Journal of Environmental Research and Public Health. 2011; 8(9):3528-52. [DOI:10.3390/ijerph8093528] [PMID] [PMCID]

[6] Kim Y, Sohn D, Choi SM. Cultural difference in motivations for using social network sites: A comparative study of American and Korean college students. Computers in Human Behavior. 2011; 27(1):365-72. [DOI:10.1016/j.chb.2010.08.015]

[7] Masters K. Social networking addiction among health sciences students in Oman. Sultan Qaboos University Medical Journal. 2015; 15(3):e357-63. [DOI:10.18295/squmj.2015.15.03.009] [PMID] [PMCID]

[8] Griffiths MD. Social networking addiction: Emerging themes and issues. Journal of Addiction Research \& Therapy. 2013; 4(5):38-47. [DOI:10.4172/2155-6105.1000e118]

[9] Doosti Irani A, Bagheri Amiri F, Khajehkazemi R, Mostafavi E. [Prevalence of Internet addiction among students and graduates of epidemiology, clinical sciences, and basic sciences in Iran: A cross-sectional study (Persian)]. Iranian Journal of Epidemiology. 2017; 13(1):14-21. http:/ /irje.tums.ac.ir/article-1-5687-en.html

[10] Tang CSK, Koh YYW. Online social networking addiction among college students in Singapore: Comorbidity with behavioral addiction and affective disorder. Asian Journal of Psychiatry. 2017; 25:175-8. [DOI:10.1016/j.ajp.2016.10.027] [PMID]

[11] Forghani MM, Mohajeri R. [The relationship between the use of figurative communication networks and the changes in the life style of youth (Persian)]. New Media Studies. 2018; 4(13):259-92. [DOI:10.22054/CS.2018.24690.293]

[12] Hajizadeh Meymandi M, Vakili Ghasemabad S, Mirmongereh A. [A survey of the relationship between socio-psychological factors and internet addiction (Case study: Girl students of Yazd University) (Persian)]. Journal of Woman in Culture Arts. 2017; 8(4):473-92. [DOI:10.22059/JWICA.2016.61790]

[13] Guraya SY. The usage of social networking sites by medical students for educational purposes: A meta-analysis and systematic review. North American Journal of Medical Sciences. 2016; 8(7):268-78. [DOI:10.4103/1947-2714.187131] [PMID] [PMCID]
[14] Farrell JM, Reiss N, Shaw IA. The schema therapy clinician's guide: a complete resource for building, group, and compilation therapy for schematic mentality [M. Hedayati, A. Samimi, Persian trans]. Tehran: Arjomand; 2017. http://opac.nlai.ir/opac-prod/ bibliographic/4498111

[15] Shajari F, Sohrabi F, Jomehri F. Relationship between early maladaptive schema and internet addiction: A cross-sectional study. Asian Journal of Pharmaceutical Research and Health Care. 2016 8(3):84-91. [DOI:10.18311/ajprhc/2016/4334]

[16] La Barbera D, La Paglia F, Valsavoia R. Social network and addiction. Studies in Health Technology and Informatics. 2009; 144:33-6. [PMID]

[17] Shayegh S, Azad H, Bahrami H. [On the relationship between internet addiction and personality traits in adolescents in Tehran (Persian)]. Journal of Fundamentals of Mental Health. 2009; 11(42):149-58. [DOI:10.22038/JFMH.2009.1637]

[18] Landers RN, Lounsbury JW. An investigation of big five and narrow personality traits in relation to internet usage. Computers in Human Behavior. 2006; 22(2):283-93. [DOI:10.1016/j. chb.2004.06.001]

[19] Ahlan AR, Balogun NA. Internet usage and personality traits: finding relationship in learning institution. Transnational Journal of Science and Technology. 2013; 3(3):1-13. http:/ / www.tjournal. org/tjst_march_2013/01.pdf

[20] Tabatabaie TS, Tahan M. [The role of Internet addiction on the quality of life of nursing students of Islamic Azad University Ghaenat (Persian)]. Journal of Zabol Medical School. 2019; 2(2):728. http://jzms.ir/index.php/JZMS/article/view/134

[21] Hakak SM, Kazemi Sh, Kakavand AR. [The association between childhood traumas and dissociative experiences with Internet addiction in students of International University of Imam Khomeiny (Persian)]. Journal of Psychological Studies. 2019; 15(1):23-40. [DOI:10.22051/PSY.2018.17767.1509]

[22] Mamashli L, Barani F, Hoijati H, Aghazi N, Hekmatipour N. [Assessment the rate of Internet addiction and mobile phone damage in nursing students of Azad University, Ali Abad Katoul, 2016 (Persian)]. Iranian Journal of Nursing Research. 2019; 14(2):15-22. http://ijnr.ir/article-1-2152-en.html

[23] Faghanpour Ganii M. [The mediating role of early maladaptive schemas in attachment styles and addiction to mobile social networks in students (Persian)]. The Journal of New Advances in Behavioral Sciences. 2018; 3(22):18-37. http://ijndibs.com/browse. php?a_id=227\&sid=1\&slc_lang=fa

[24] Ghiasy M, Molavi H, Neshat doost HT, Salavati T. [Validation of Yang schema questionnaire (The third edition of the short form) in two groups of normal and mentally impaired people in Tehran (Persian)]. Journal Psychological Knowledge. 2012 1(3):64-72. https:/ / psychac.scu.ac.ir/article_11692.html?lang=fa

[25] Khajeahmadi M, Pooladi Sh, Bahreini M. [Design and assessment of psychometric properties of the addiction to mobile questionnaire based on social networks (Persian)]. Iranian Journal of Psychiatric Nursing. 2017; 4(4):43-51. http://ijpn.ir/article1-884-en.htm

[26] Beheshtian M. [Comparing early maladaptive schemas of healthy female students and those addicted to the Internet (Persian)]. Women Studies. 2013; 4(8):61-71. http://womenstudy. ihcs.ac.ir/article_1072.html 
[27] Malcolm M, Frost H, Cowie J. Loneliness and social isolation causal association with health-related lifestyle risk in older adults: A systematic review and meta-analysis protocol. Systematic Reviews. 2019; 8(1):48. [DOI:10.1186/s13643-019-0968-x] [PMID] [PMCID]

[28] Weiner JL, Karkhanis AN, Rau A, Skelly MJ, Jones SR. SY172: Adolescent social isolation as a model of alcohol addiction vulnerability. Alcohol and Alcoholism. 2015; 50(Suppl_1):i20. [DOI:10.1093/alcalc/agv076.71]

[29] Wafae IS, Tarik Gh, Ahmed A, Fatima-Zahrae A, Khadija K, Khaoula M. Measurement of early maladaptive schemas in heroin addicts treated with methadone in north of Morocco. European Journal of Investigation in Health Psychology and Education, 2018; 8(3):185-96. https://www.mdpi.com/2254-9625/8/3/185 